\title{
JNT Special Issue MDPD Congress, Berlin 2012
}

\author{
Amos D. Korczyn · Heinz Reichmann
}

Published online: 21 March 2013

(c) Springer-Verlag Wien 2013

The field of Parkinson's disease is quite dynamic. For one century and a half, it was considered to be almost exclusively a movement disorder, while non-motor features have received meager if any attention.

The first meetings specifically highlighting these forgotten areas were held in 1993 in Amsterdam and Jerusalem, and they paved the way to an astonishing expansion in the field.

The present volume includes selected presentations from the Eighth International Congress on Mental Dysfunctions and other non-motor features in Parkinson's disease and related disorders which took place in Berlin with over 800 participants. The topics, as seen in this volume, extended across the field to include mental, autonomic, sensory, and other manifestations, in Parkinson's disease as well as other movement disorders, with emphasis on the diagnosis and therapy.

Following the success of the 2013 Congress in Seoul, the next meeting, the Tenth International Congress on Mental Dysfunctions and other non-motor features in Parkinson's disease and related disorders will take place in Nice, France, on 4-7 December 2014, with an exciting new program. We hope to see many of you there.

\footnotetext{
A. D. Korczyn $(\bowtie)$

Department of Neurology, Tel Aviv University,

69978 Ramat Aviv, Israel

e-mail: amoskor@tau.ac.il

H. Reichmann

Department of Neurology, Technische Universitaet Dresden,

Fetscherstrasse 74, 01307 Dresden, Germany

e-mail: Heinz.Reichmann@uniklinikum-dresden.de
} 